\title{
CLASH: EXTREME EMISSION-LINE GALAXIES AND THEIR IMPLICATION ON SELECTION OF HIGH-REDSHIFT GALAXIES
}

\author{
Xingxing Huang ${ }^{1,2}$, Wei Zheng ${ }^{2}$, JunXian Wang ${ }^{1}$, Holland Ford ${ }^{2}$, Doron Lemze ${ }^{2}$, John Moustakas $^{3}$, Xinwen Shu $^{1,4}$, \\ Arjen Van der Wel ${ }^{5}$, Adi Zitrin ${ }^{6,20}$, Brenda L. Frye ${ }^{7}$, Marc Postman $^{8}$, Matthias BartelmanN ${ }^{9}$, Narciso Benítez $^{10}$, \\ Larry Bradley ${ }^{8}$, Tom Broadhurst ${ }^{11,12}$, Dan CoE $^{8}$, Megan Donahue $^{13}$, Leopoldo Infante ${ }^{14}$, Daniel Kelson ${ }^{15}$, \\ Anton Koekemoer ${ }^{8}$, Ofer Lahav ${ }^{16}$, Elinor Medezinski ${ }^{2}$, LeOnidas Moustakas $^{15}$, Piero Rosati ${ }^{17}$, \\ Stella Seitz ${ }^{18}$, And Keitchi Umetsu ${ }^{19}$ \\ ${ }^{1}$ CAS Key Laboratory for Research in Galaxies and Cosmology, Department of Astronomy, University of Science and Technology of China, \\ Hefei, Anhui 230026, China; e-mail:hxx@ mail.ustc.edu.cn \\ 2 Department of Physics and Astronomy, Johns Hopkins University, 3400 North Charles Street, Baltimore, MD 21218, USA \\ ${ }^{3}$ Department of Physics and Astronomy, Siena College, 515 Loudon Road, Loudonville, NY 12211, USA \\ ${ }^{4}$ CEA Saclay, DSM/Irfu/Service d'Astrophysique, Orme des Merisiers, F-91191 Gif-sur-Yvette Cedex, France \\ ${ }_{5}^{5}$ Max-Planck Institute for Astronomy, Königstuhl 17, D-69117, Heidelberg, Germany \\ ${ }^{6}$ Cahill Center for Astronomy and Astrophysics, California Institute of Technology, MS 249-17, Pasadena, CA 91125, USA \\ ${ }^{7}$ Steward Observatory/Department of Astronomy, University of Arizona, 933 North Cherry Avenue, Tucson, AZ 85721-0065, USA \\ ${ }^{8}$ Space Telescope Science Institute, 3700 San Martin Drive, Baltimore, MD 21208, USA \\ ${ }^{9}$ Leiden Observatory, Leiden University, P. O. Box 9513, 2300 RA Leiden, The Netherlands \\ ${ }^{10}$ Instituto de Astrofísica de Andalucía (CSIC), C/Camino Bajo de Huétor 24, Granada E-18008, Spain \\ ${ }^{11}$ Department of Theoretical Physics, University of Basque Country UPV/EHU E-Bilbao, Spain \\ ${ }^{12}$ IKERBASQUE, Basque Foundation for Science, Bilbao, Spain \\ ${ }^{13}$ Department of Physics and Astronomy, Michigan State University, East Lansing, MI 48824, USA \\ ${ }^{14}$ Departamento de Astronoía y Astrofísica, Pontificia Universidad Católica de Chile, V. Mackenna 4860 Santiago 22, Chile \\ ${ }^{15}$ Observatories of the Carnegie Institution of Washington, Pasadena, CA 91101, USA \\ ${ }^{16}$ Department of Physics and Astronomy, University College London, Gower Street, London WCIE 6 BT, UK \\ ${ }^{17}$ Institute of Astronomy and Astrophysics, Academia Sinica, P.O. Box 23-141, Taipei 10617, Taiwan \\ ${ }^{18}$ Universitäts-Sternwarte, München, Scheinerstraße 1, D-81679 München, Germany \\ ${ }^{19}$ Institut für Theoretische Astrophysik, ZAH, Albert-Ueberle-Straße 2, D-69120 Heidelberg, Germany \\ Received 2014 July 6; accepted 2014 December 22; published 2015 February 25
}

\begin{abstract}
We utilize the Cluster Lensing And Supernova survey with Hubble observations of 25 clusters to search for extreme emission-line galaxies (EELGs). The selections are carried out in two central bands: F105W $\left(Y_{105}\right)$ and F125W $\left(J_{125}\right)$, as the flux of the central bands could be enhanced by the presence of [O III] $\lambda \lambda 4959,5007$ at redshifts of $\sim 0.93-1.14$ and 1.57-1.79, respectively. The multiband observations help to constrain the equivalent widths (EWs) of emission lines. Thanks to cluster lensing, we are able to identify 52 candidates down to an intrinsic limiting magnitude of 28.5 and to a rest-frame [O III] $\lambda \lambda 4959,5007 \mathrm{EW}$ of $\simeq 3700 \AA$. Our samples include a number of EELGs at lower luminosities that are missed in other surveys, and the extremely high EW can only be found in such faint galaxies. These EELGs can mimic a dropout feature similar to that of high-redshift galaxies and contaminate the color-color selection of high-redshift galaxies when the signal-to-noise ratio is limited or the band coverage is incomplete.
\end{abstract}

Key words: galaxies: formation - galaxies: high-redshift - galaxies: photometry

\section{INTRODUCTION}

The presence of extremely strong emission lines such as the [O $\mathrm{III}] \lambda \lambda 4959,5007$ and $\mathrm{H} \alpha \lambda 6563$ emission lines is one of the prominent spectral features in star-forming galaxies. Recently, a considerable number of star-forming galaxies with extraordinarily strong [O III] $\lambda \lambda 4959,5007$ (Straughn et al. 2009; van der Wel et al. 2011; Atek et al. 2011; Smit et al. 2014) or $\mathrm{H} \alpha \lambda 6563$ (Shim et al. 2011; Shim \& Chary 2013) lines have been found. While some of these galaxies are identified spectroscopically (Erb et al. 2006; Atek et al. 2011; Frye et al. 2012), the majority of them are found from broadband photometry with a significant flux excess in one or more bands. Utilizing the Hubble Space Telescope/Wide Field Camera 3 (HST/WFC3) observations of the Cosmic Assembly NearIR Deep Extragalactic Legacy Survey (CANDELS; Grogin et al. 2011; Koekemoer et al. 2011), van der Wel et al. 2011 (VDW11 hereafter) identified an abundant population of

\footnotetext{
${ }^{20}$ Hubble Fellow.
}

extreme emission-line galaxies (EELGs) at redshift $z \sim 1.7$. In some cases, the rest-frame equivalent widths (EWs) of such strong emission lines reach $2000 \AA$ or even higher.

Extremely strong emission lines can affect the spectralenergy-distribution (SED) fitting of broadband photometry (Schaerer \& de Barros 2009; Atek et al. 2011; Shim et al. 2011; Labbé et al. 2010, 2012; Stark et al. 2013; Fumagalli et al. 2012). Their contributions may mimic the spectral feature of the Lyman break in high-redshift galaxies. It is therefore possible that some high-redshift Lyman break galaxies (LBGs) may actually be low-redshift EELGs when the wavelength coverages or depths are limited. Recently, the search for LBGs has reached $z>9$, and HST plays the major role in this redshift range with the WFC3/IR instrument (Bouwens et al. 2011; Zheng et al. 2012b; Coe et al. 2013; Ellis et al. 2013; Oesch et al. 2013). UDFj-39546284 was first detected in the Hubble Ultra Deep Field (HDF09) with an $H_{160}$ band detection alone (Bouwens et al. 2011). The decrement between the F160W and $\mathrm{F} 125 \mathrm{~W}$ bands is larger than $2 \mathrm{mag}$, thus suggestive of a $z \simeq 10$ candidate. Follow-up observations of the HUDF12 
(GO 12498: Ellis et al. 2013; Koekemoer et al. 2013) and CANDELS program (Grogin et al. 2011; Koekemoer et al. 2011) confirm that this substantial break in the SED is actually between the F160W and F140W bands (Ellis et al. 2013; Bouwens et al. 2013), implying an even higher redshift. Brammer et al. (2013) analyzed deep WFC3 grism observations of UDFj-39546284 and found a $2.7 \sigma$ detection of an emission line at $1.599 \mu \mathrm{m}$. In the deep Keck observation, Capak et al. (2013) also found a $2.2 \sigma$ peak at the same wavelength. Both spectra suggest that UDFj39546284 could be a strong [O III] $\lambda 5007$ emitter at $z \sim 2.19$ or a strong [O II] $\lambda 3727$ emitter at $z \sim 3.29$. Current deep nearinfrared observations are unlikely to determine the nature of this candidate. The presence of UDFj-39546284 suggests that the possible contamination due to EELGs at lower redshift should be reexamined.

In this paper, we report the search for EELGs at two redshift ranges in the Cluster Lensing And Supernova survey with Hubble (CLASH) cluster fields to estimate the contamination to the selections of LBGs. The CLASH program (Postman et al. 2012 ) is a 16 band survey of 25 clusters between 0.2 and $1.6 \mu \mathrm{m}$. The nominal limiting magnitude in the F160W band

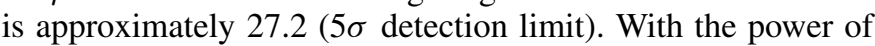
cluster lensing, some of the targets can reach an intrinsic $\mathrm{AB}$ magnitude $\sim 29 \mathrm{mag}$, similar to that of $z \sim 10$ galaxies in the Hubble Ultra Deep Field.

Throughout this paper, EW refers to the total of [O III] $\lambda \lambda 4959,5007$ and $\mathrm{H} \beta \lambda 4861$ in the rest frame, unless otherwise specified. Magnitudes are calculated in the AB system. Errors are computed at $1 \sigma$. We adopt a flat cosmology with $\Omega_{\lambda}=0.7$, $\Omega_{M}=0.3$ and $H_{0}=70 \mathrm{~km} \mathrm{~s}^{-1} \mathrm{Mpc}^{-1}$.

\section{DATA}

\subsection{Data Reduction}

The CLASH data were obtained with three HST cameras: Advanced Camera for Surveys (ACS)/WFC, WFC3/IR, and WFC3/UVIS. All 25 clusters have now been observed. The images and catalogs are processed with APLUS (Zheng et al. 2012a), which is an automatic pipeline modified from the APSIS package (Blakeslee et al. 2003) with an enhanced capability of processing WFC3 data and aligning them with the ACS data. APLUS processes the calibrated images from the HST instrument pipelines, namely, the $f c$ images for ACS (corrected for the detector's charge transfer efficiency) and the $f t$ images for WFC3/IR. Recently, APLUS has been updated so that images of individual exposure are aligned using DrizzlePac (Gonzaga et al. 2012), and the accuracy can achieve $1 / 5$ pixel $\left(\sim 0^{\prime \prime} .015\right)$ or better.

In the APLUS process, images of different filter bands and cameras were further aligned, resampled, and combined, with a common pixel scale of 0.065 . We created detection images from the weighted sum of ACS/WFC and WFC3/IR images and ran SExtractor (Bertin \& Arnouts 1996) in a dual mode for all 16 bands. mag_iso were chosen in the color selections. We also verified the photometry by comparing with public catalogs, ${ }^{21}$ which were processed with a modified version of the Mosaicdrizzle pipeline (Koekemoer et al. 2003, 2011), and no systematic deviation was found between the two pipelines. In this paper, we focus on two ACS/WFC filters (F814W, F850LP) and five WFC3/IR filters (F105W, F110W, F125W, F140W, $\mathrm{F} 160 \mathrm{~W})$, which are hereafter called the $I_{814}, Z_{850}, Y_{105}, Y J_{110}$, $J_{125}, J H_{140}$, and $H_{160}$ bands.

\footnotetext{
21 http://archive.stsci.edu/prepds/clash/
}

\subsection{Sample Selection}

A color-color selection has been successfully used for identifying EELGs in VDW11. We carried out two selections with two sets of filter bands. First, we follow the selection criteria of VDW11, namely,

$$
J_{125}-I_{814}<-0.44-\sigma \wedge J_{125}-H_{160}<-0.44-\sigma .
$$

In the other selection, we use the $Y_{105}$ band as the central band, namely,

$$
Y_{105}-I_{814}<-0.44-\sigma \wedge Y_{105}-H_{160}<-0.44-\sigma
$$

where the $\sigma$ refers to the $1 \sigma$ error of the color. We also require that the three bands in each selection are detected above $3 \sigma$ to ensure good EW measurements. After these preliminary selections (Figure 1), we check the images and photometry of the WFC3/IR bands for each object. Sources contaminated by cosmic-ray events, nearby bright sources, and detector-edge effects are excluded. We build two samples with 40 and 12 candidates named as the "J" sample and "Y" sample. Note that the color excess of $0.44 \mathrm{mag}$ in $J_{125}$ and $Y_{105}$ corresponds to a rest-frame EW of about $600 \AA$. The composite color images of these galaxies are shown in Figure 2. We include the apparent angular sizes, which are measured through full width at halfmaximum (FWHM) by SExtractor in Tables 1 and 2.

\subsection{Redshift Estimation}

To illustrate the boosting effect in different bands and different redshifts, we simulate model spectra with a simple powerlaw continuum plus emission lines and obtain the observed magnitudes using the throughputs of $H S T$ filters (lower panel in Figure 3). The index of the power-law continuum is fixed at $\beta=2$, which is defined as $F_{\lambda} \sim \lambda^{-\beta}$. Such a continuum is a constant in different wavelengths with $\mathrm{AB}$ magnitude system and is set to $28 \mathrm{mag}$. In the model, we choose metal-line lists from galaxies with subsolar metallicity of $Z=0.2 Z_{\odot}=0.004$ in Anders et al. (2003) and only include emission lines with relative line intensities $F_{\text {line }} / F_{H_{\beta}}$ larger than 0.1 . The $H_{\alpha}$ line is included by assuming the ratio $H_{\alpha} / H_{\beta}=2.86$ from case $\mathrm{B}$ recombination (Storey \& Hummer 1995).

In the model, the $\operatorname{EW}([\mathrm{O} I I I] ~ \lambda 5007)$ is set to $2000 \AA$. The simulated magnitudes and colors are shown with thick blue lines in Figure 4. Based on this model, those EELGs in the redshift ranges $\sim 1.57-1.79$ (the J sample) and $\sim 0.93-1.14$ (the Y sample) are selected (gray regions in Figure 4). The redshift ranges would not change significantly if we use a different EW in the model. The upper panel of Figure 3 shows the wavelength ranges where the strongest emission lines, $\mathrm{H} \alpha \lambda$ 2563 , [O III] $\lambda 5007$, and [O II] $\lambda 3727$ impact the observed flux. For the Y sample, the [O II] $\lambda 3727$ falls into the $I_{814}$ band; thus EELGs with stronger emission lines can be selected.

The spectral slopes of star-forming galaxies have been shown with a $1 \sigma$ dispersion of 0.4 (Bouwens et al. 2009). In the second model, we take into consideration the effect of spectral slopes and vary them between $\beta=1.5$ and 2.5. The effect is shown in a blue shadow region in the right panel of Figure 4 . The changes in spectral slope would affect the color excess lower than 0.25 mag. Some EELGs with blue slopes will not be selected. However, as the continuum is calculated with the average of the two bands at different wavelengths, the change of slope is not a problem in the following EW estimates. 

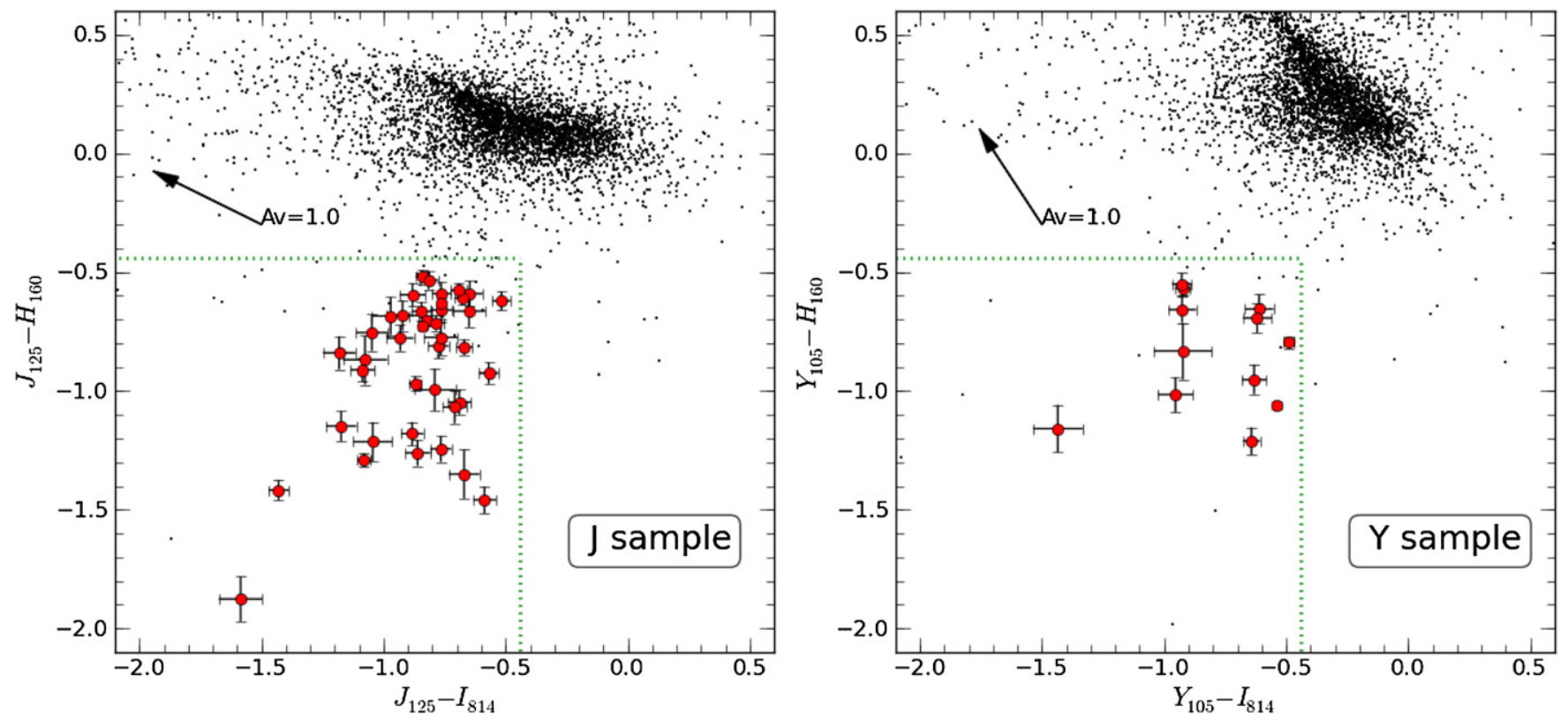

Figure 1. Color-color diagrams of the EELG candidate selection. The red circles with error bars mark the selected EELGs, and the black points mark all objects in the CLASH fields. The regions separated by dashed lines represent our selection criteria. The black arrows illustrate the effect of dust attenuation, using the formula from Cardelli et al. (1989).
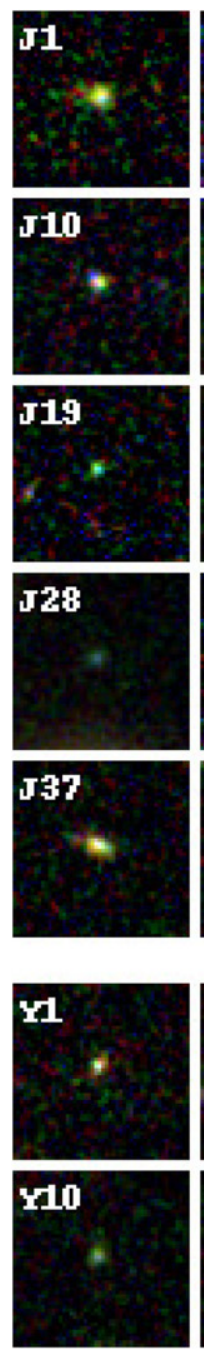
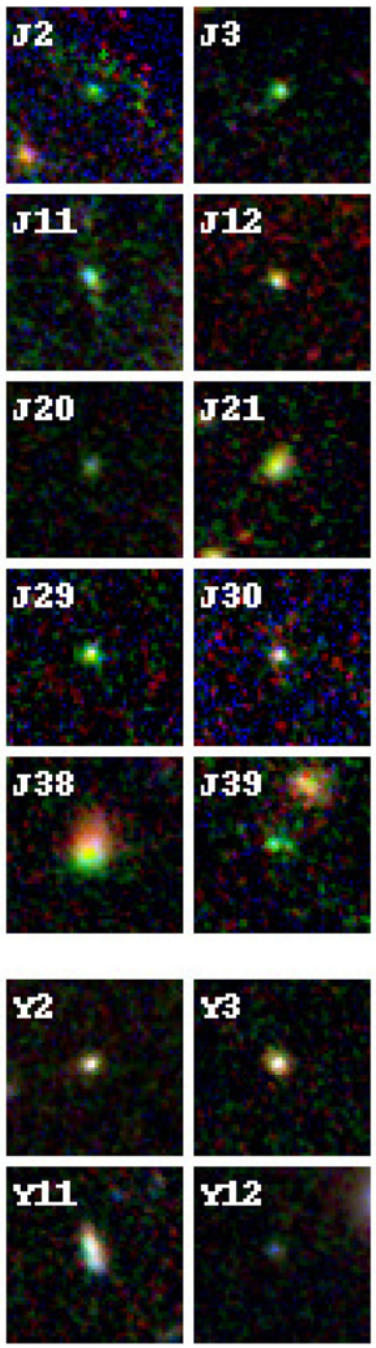
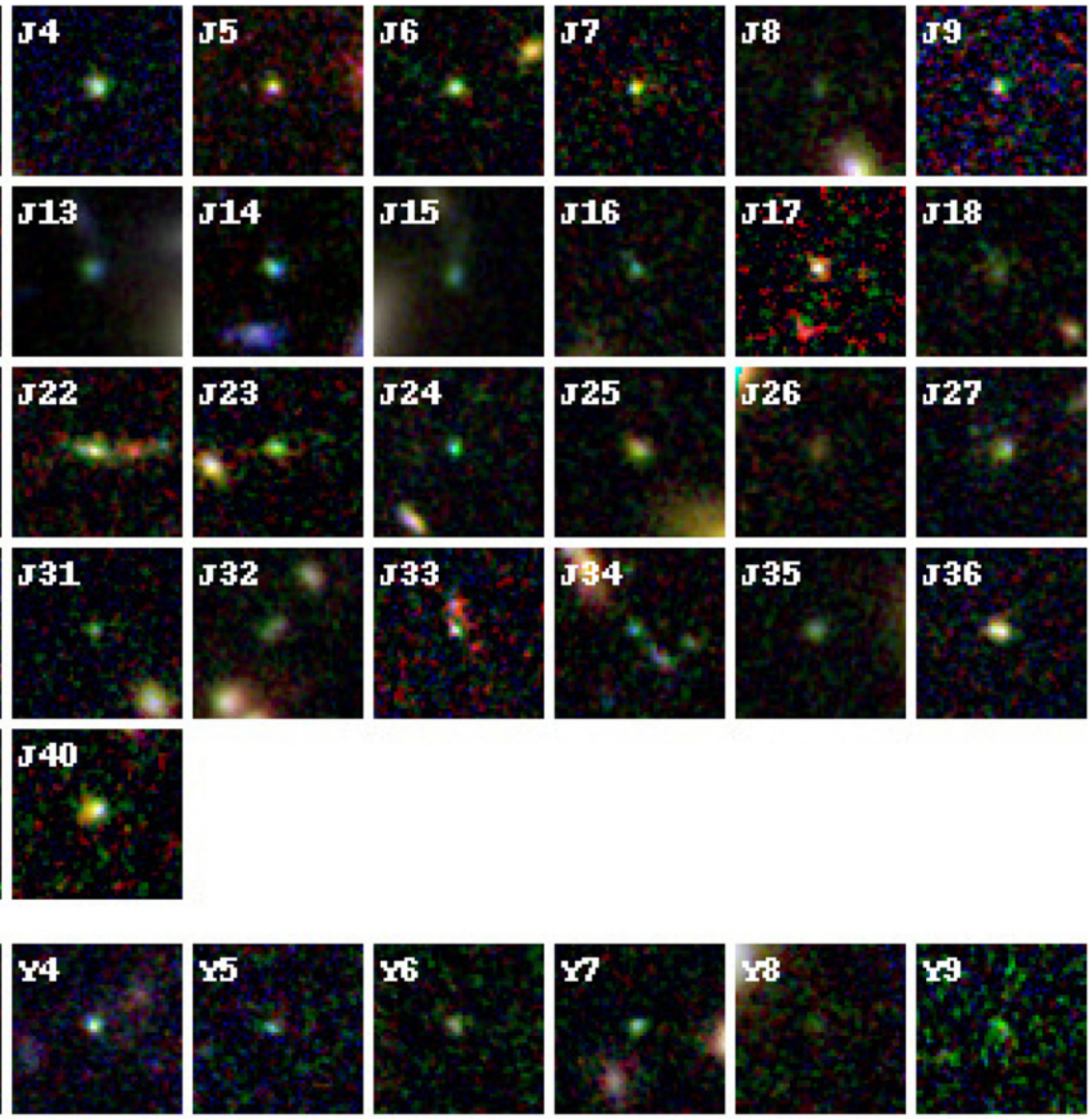

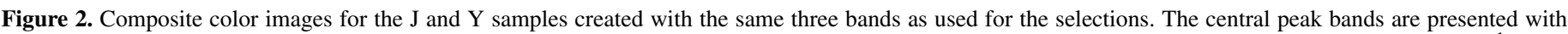
green color, while the continuum bands are presented with blue and red colors. The width of each stamp image is 50 pixels, and the pixel size is 0 ' $^{\prime} 065$ pixel ${ }^{-1}$. 


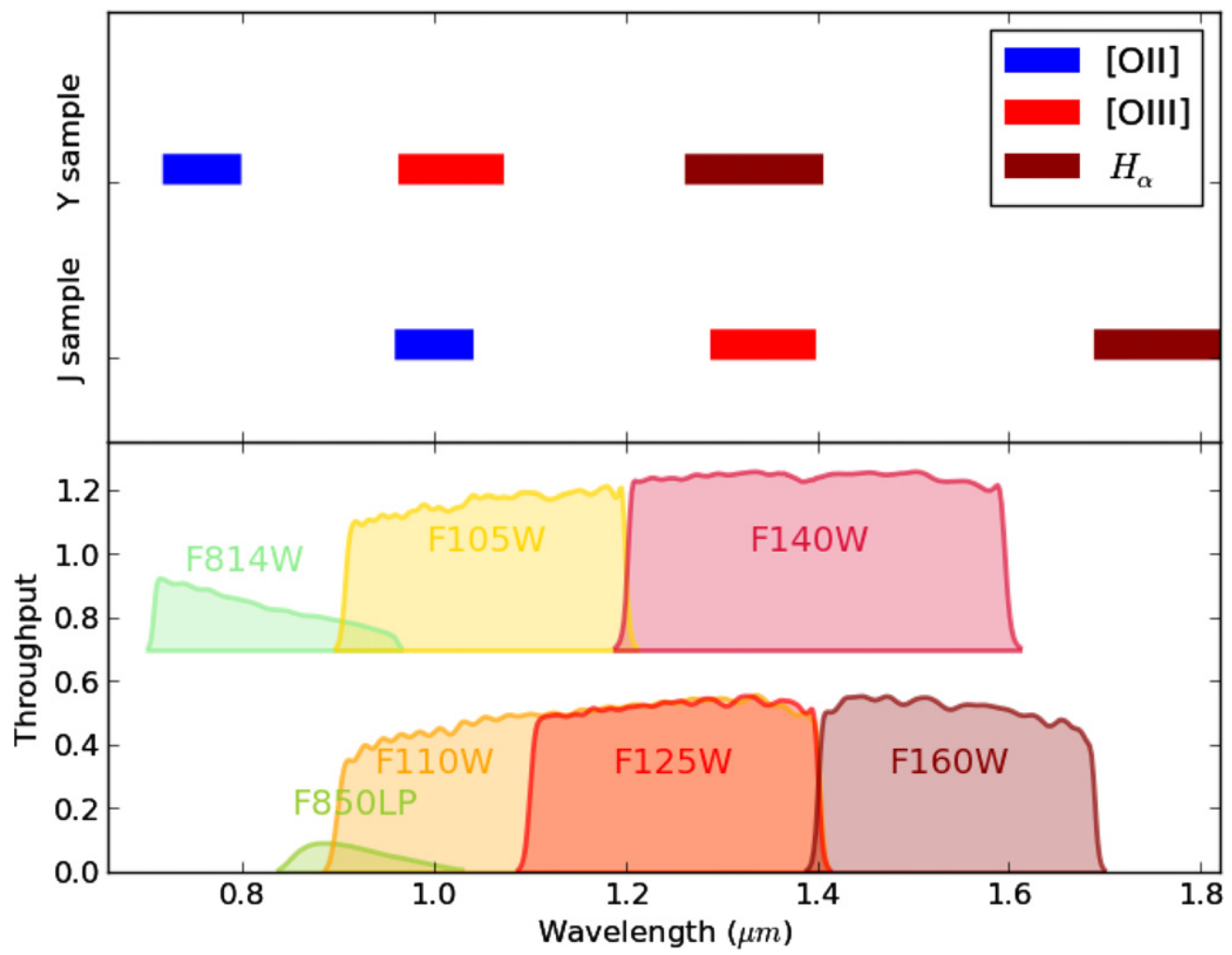

Figure 3. Wavelength ranges of strong emission lines in our J and Y samples. The top panel shows the wavelength ranges for [O II], [O III], and $H_{\alpha}$ with the redshifts of the $\mathbf{J}$ sample and the Y sample. The lower panel shows the throughputs of the HST filters. There are a total of 16 filters used in the CLASH observations, but only the red filters related to strong emission lines are shown here.
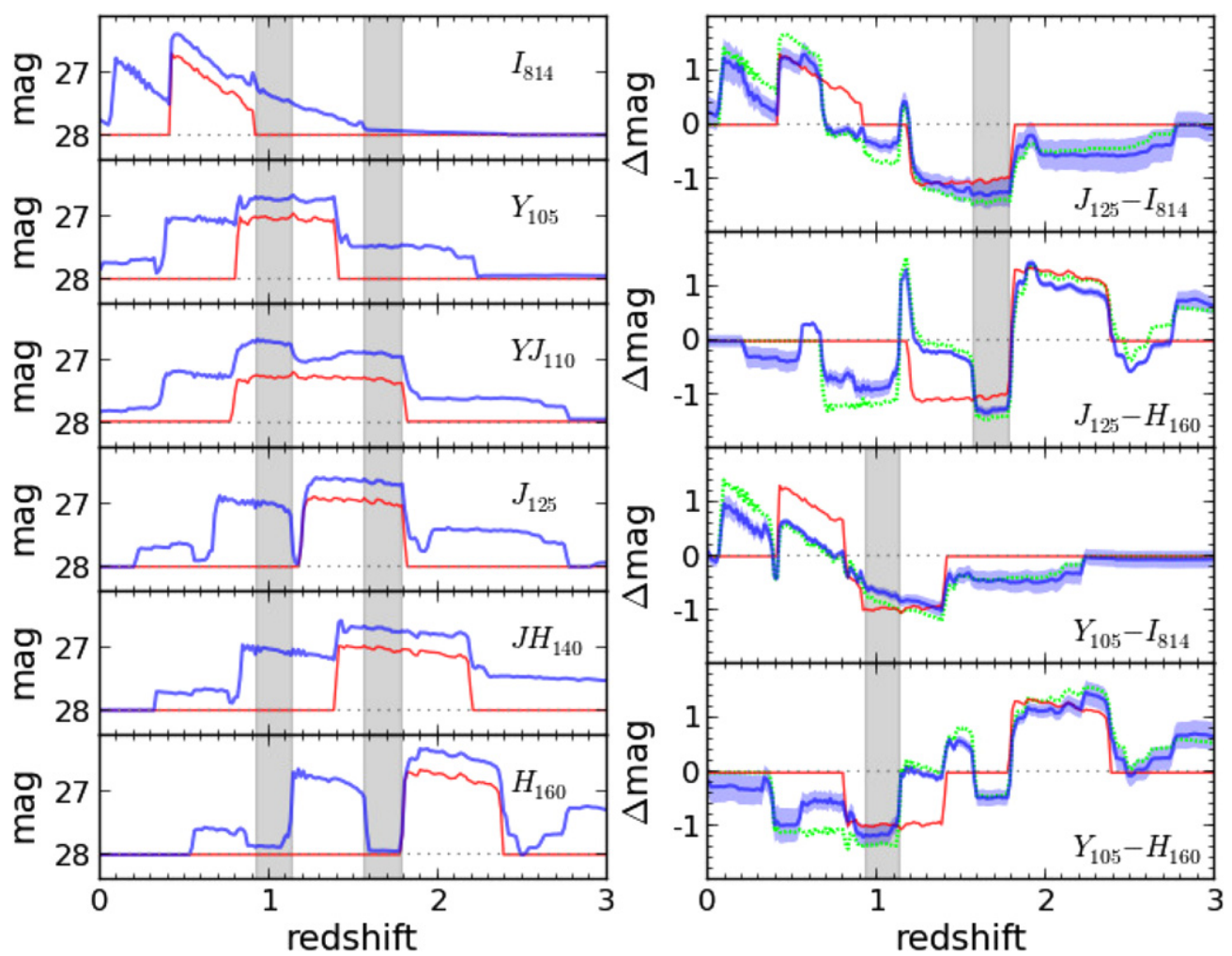

Figure 4. Effect of strong emission lines on the photometry as a function of redshift, estimated with our spectral model. The spectral model represents the spectrum of a star-forming galaxy, which consists of a power-law spectrum and strong emission lines with the flux ratios from Anders et al. (2003). In the left panels, the photometry of the six bands used in the EW calculations is plotted vs. the redshift. In the right panels, the colors used for our selections are plotted vs. the redshift (upper: the J sample, lower: the Y sample). In the spectral model, the two sets of emission-line ratios for metallicity $Z=0.2 Z_{\odot}$ and $Z=0.02 Z_{\odot}$ are considered, which are shown with blue lines and green dotted lines. The blue shadows around the blue lines in the right panels present the influence of the spectral slope, which changes from 1.5 to 2.5 . The contributions of the $[\mathrm{O}$ III] $\lambda \lambda 4959,5007$ lines are shown with red lines. Our two color-color selections are quite effective for selections of EELGs in the two redshift ranges (0.93-1.14 and 1.57-1.79) marked with gray boxes. 
Table 1

EELG in the Y Sample

\begin{tabular}{|c|c|c|c|c|c|c|c|c|c|c|c|c|c|}
\hline EELG & Cluster & $\begin{array}{c}\text { R.A. } \\
\text { deg } \\
(3) \\
\end{array}$ & $\begin{array}{c}\text { Decl. } \\
\text { deg } \\
(4) \\
\end{array}$ & $\begin{array}{c}I_{814} \\
\mathrm{AB} \\
(5) \\
\end{array}$ & $\begin{array}{c}J_{125} \\
\mathrm{AB} \\
(6) \\
\end{array}$ & $\begin{array}{c}H_{160} \\
\mathrm{AB} \\
(7) \\
\end{array}$ & $\begin{array}{c}\mathrm{EW}_{Y J 110} \\
\AA \\
(8) \\
\end{array}$ & $\begin{array}{c}\mathrm{EW}_{J 125} \\
\AA \\
(9) \\
\end{array}$ & $\begin{array}{c}\mathrm{EW}_{J H 140} \\
\AA \\
(10) \\
\end{array}$ & (11) & (12) & $\begin{array}{c}\text { FWHM } \\
\operatorname{arcsec} \\
(13) \\
\end{array}$ & $\begin{array}{c}\log (M) \\
M_{\odot} \\
(14) \\
\end{array}$ \\
\hline $\mathrm{J} 1$ & a1423 & 179.33289 & 33.60424 & $25.88 \pm 0.06$ & $25.37 \pm 0.05$ & $25.99 \pm 0.07$ & $640 \pm 181$ & $774 \pm 160$ & $842 \pm 188$ & $1.4 \pm 0.1$ & 1.6 & 0.37 & $7.8 \pm 0.2$ \\
\hline$J 2^{\mathrm{a}}$ & a209 & 22.95528 & -13.60011 & $27.92 \pm 0.21$ & $26.33 \pm 0.10$ & $28.21 \pm 0.32$ & $3145 \pm 1169$ & $4259 \pm 1354$ & $3976 \pm 1270$ & $2.1 \pm 0.4$ & 1.9 & 0.29 & $6.7 \pm 6$ \\
\hline $\mathrm{J} 4$ & a209 & 22.98267 & -13.60450 & $25.91 \pm 0.08$ & $25.07 \pm 0.05$ & $25.73 \pm 0.07$ & $983 \pm 260$ & $1150 \pm 219$ & $1016 \pm 251$ & $1.7 \pm 0.1$ & 1.3 & 0.28 & \\
\hline J5 & a383 & 42.01418 & -3.54037 & $26.80 \pm 0.11$ & $25.92 \pm 0.06$ & $26.51 \pm 0.09$ & $1569 \pm 362$ & $1149 \pm 269$ & $1762 \pm 382$ & $2.0 \pm 0.2$ & 1.7 & 0.27 & $6.7 \pm 0.1$ \\
\hline J6 & 383 & 42.00668 & -3.54478 & $26.25 \pm 0.07$ & $25.68 \pm 0.05$ & $26.61 \pm 0.10$ & $1215 \pm 271$ & $1111 \pm 214$ & $983 \pm 264$ & $2.4 \pm 0.1$ & 1.2 & 0.26 & \\
\hline J7 & a383 & 42.00755 & -3.54529 & $27.10 \pm 0.12$ & $26.45 \pm 0.08$ & $27.12 \pm 0.13$ & $1272 \pm 427$ & $984 \pm 323$ & $1255 \pm 433$ & $2.2 \pm 0.2$ & 1.2 & .30 & $6.9 \pm 0.2$ \\
\hline J11 & m0329 & 2.43664 & -2.20187 & $26.28 \pm 0.09$ & $25.64 \pm 0.06$ & $26.23 \pm 0.09$ & $1218 \pm 339$ & $946 \pm 245$ & $1041 \pm 296$ & $2.5 \pm 0.1$ & 2.8 & 0.77 & $6.9 \pm 0$ \\
\hline $\mathrm{J} 12$ & 0329 & 52.42710 & -2.20908 & $26.39 \pm 0.09$ & $25.70 \pm 0.06$ & $26.75 \pm 0.12$ & $1720 \pm 393$ & $1401 \pm 297$ & $1522 \pm 352$ & $2.0 \pm 0.1$ & 2.9 & & ( \\
\hline $\mathrm{J} 13$ & $\mathrm{~m} 0416$ & 64.04457 & -24.07828 & $24.65 \pm 0.04$ & $23.83 \pm 0.02$ & $24.53 \pm 0.04$ & $801 \pm 127$ & $1198 \pm 110$ & $1052 \pm 126$ & $1.7 \pm 0.1$ & 2.0 & 0.25 & $7.7 \pm 0$ \\
\hline J14 & 0416 & 64.04506 & -24.08220 & $26.51 \pm 0.11$ & $25.58 \pm 0.06$ & $26.35 \pm 0.11$ & $1569 \pm 417$ & $1398 \pm 327$ & $1252 \pm 368$ & $1.7 \pm 0.3$ & 1.4 & & \\
\hline J15 & m0429 & 67.39312 & -2.88310 & $24.52 \pm 0.03$ & $23.68 \pm 0.02$ & $24.40 \pm 0.03$ & $1560 \pm 122$ & $1268 \pm 98$ & $1495 \pm 119$ & $2.0 \pm 0.1$ & 2.4 & 0.72 & $7.6 \pm 0.1$ \\
\hline J16 & 0647 & 101.89789 & 70.24 & $26.75 \pm 0.06$ & $25.97 \pm 0.06$ & $26.79 \pm 0.13$ & & $1413 \pm 259$ & $1428 \pm 342$ & $1.9 \pm 0.2$ & 1.6 & 40 & $6.7 \pm 0.1$ \\
\hline J17 & 0717 & 109.36207 & 37.74810 & $26.41 \pm 0.06$ & $25.70 \pm 0.09$ & $26.76 \pm 0.12$ & $1652 \pm 350$ & $1356 \pm 312$ & $1309 \pm 279$ & $2.4 \pm$ & 2.1 & .30 & $6.8 \pm 0.1$ \\
\hline J18 & 0744 & & & $27.13 \pm 0.12$ & $5.95 \pm 0.08$ & $26.79 \pm 0.14$ & $1630 \pm 554$ & $2056 \pm 501$ & $1203 \pm 444$ & $2.8 \pm$ & 1. & & $6.6 \pm 0.1$ \\
\hline J24 & & & -8.8 & $7 \pm 0.08$ & $8 \pm 0.06$ & $27.34 \pm 0.26$ & $\ldots$ & $9 \pm 302$ & $34 \pm$ & $2.2 \pm$ & 2. & & $6.7 \pm($ \\
\hline J25 & 1311 & 7.74210 & -3.16 & $25.55 \pm 0.05$ & $24.71 \pm 0.03$ & $25.23 \pm 0$ & $\ldots$ & $1161 \pm 150$ & $1233 \pm 195$ & $2.0 \pm 0.1$ & 1.2 & 0.25 & $7.5 \pm($ \\
\hline J26 & & & -3.1 & 2 & $6.22 \pm 0.09$ & $26.91 \pm 0$ & & $61 \pm$ & $86 \pm$ & & & & $6.9 \pm($ \\
\hline J27 & $\mathrm{m} 1311$ & 197.77126 & -3.16271 & $26.02 \pm 0.08$ & $25.25 \pm 0.06$ & $25.91 \pm 0.12$ & & $1133 \pm 250$ & $1185 \pm 320$ & $2.6 \pm 0.1$ & 1. & 45 & $7.3 \pm($ \\
\hline $\mathrm{J} 28$ & $\mathrm{~m} 1423$ & & & $.52 \pm 0.06$ & $.76 \pm 0.06$ & $27.00 \pm 0.15$ & $846 \pm 299$ & $+2 \pm 275$ & $57 \pm 308$ & $2.0 \pm$ & 2. & 0.57 & $6.8 \pm($ \\
\hline$J 29^{\mathrm{a}}$ & 1720 & 02 & 3 & $27.08 \pm 0.11$ & $25.65 \pm 0.04$ & $27.06 \pm 0.18$ & & $3145 \pm 555$ & $3648 \pm 718$ & $2.0 \pm$ & 1.1 & 0.25 & $6.6 \pm 0$ \\
\hline J30 & & & & $7.35 \pm 0.14$ & $.18 \pm 0.07$ & $27.32 \pm 0.17$ & $1209 \pm 501$ & $2207 \pm 536$ & $2191 \pm 528$ & & & & \\
\hline J31 & 1931 & 292.93714 & -26.58000 & $27.30 \pm 0.17$ & $26.50 \pm 0.10$ & $27.50 \pm 0.19$ & $1974 \pm 710$ & $1524 \pm 544$ & $1266 \pm 589$ & $2.3 \pm 0.3$ & 1.2 & 31 & $7.0 \pm($ \\
\hline $\mathrm{J} 32$ & $\mathrm{~m}$ & & -7.67390 & $25.98 \pm 0.06$ & $.16 \pm 0.05$ & $25.69 \pm 0.06$ & $454 \pm 248$ & $1162 \pm 197$ & $1274 \pm 230$ & & & & \\
\hline $\mathrm{J} 33$ & 2129 & 2.36494 & -7.70077 & $27.10 \pm 0.11$ & $26.33 \pm 0.09$ & $27.11 \pm 0.14$ & $1121 \pm 450$ & $1271 \pm 381$ & $1239 \pm 433$ & $2.0 \pm$ & & & 1 \\
\hline J34 & 212 & 282 & -7.70 & $26.74 \pm 0$ & $26.07 \pm 0.12$ & $27.42 \pm$ & $1059 \pm 376$ & $36 \pm 433$ & $611 \pm$ & $2.4 \pm$ & 1. & 0.30 & $7.1 \pm$ \\
\hline J35 & 2137 & 3 & -23.64 & $5.73 \pm 0.06$ & $.06 \pm 0$. & 25.8 & $1339 \pm 209$ & $1166 \pm 172$ & $1314 \pm$ & $1.4 \pm$ & 1 & & $7.4 \pm($ \\
\hline J36 & & & -23.6 & $25.81 \pm 0$ & $25.02 \pm 0$ & $25.74 \pm 0$ & $862 \pm 190$ & $1180 \pm 174$ & $1242 \pm$ & & 1 & 0.27 & \\
\hline J37 & 2137 & 5.06148 & -23.675 & $25.64 \pm 0.06$ & $24.95 \pm 0.04$ & $25.53 \pm 0$ & $949 \pm 174$ & $942 \pm 146$ & $1020 \pm 177$ & $2.0 \pm 0.1$ & 1 & 0.38 & $7.9 \pm($ \\
\hline J38 & & & -11.7 & $23.73 \pm 0.04$ & $2.97 \pm 0$. & & $800 \pm 49$ & & & & 1 & 56 & \\
\hline
\end{tabular}

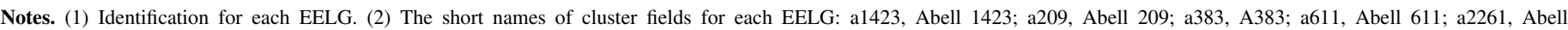

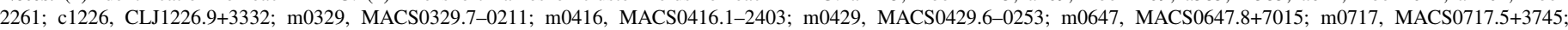

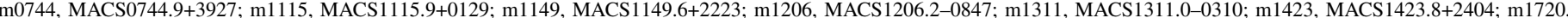

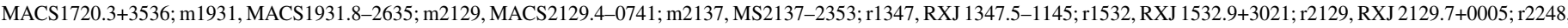

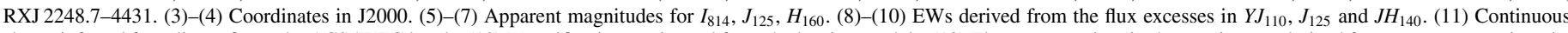

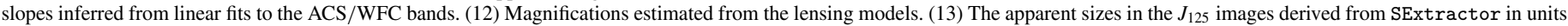
of arcseconds. (14) Stellar masses inferred from Starburst99 (Leitherer et al. 1999).

${ }^{a}$ EELGs that have error-weighted average of EWs higher than $3000 \AA$.

Table 2

EELG in the Y Sample

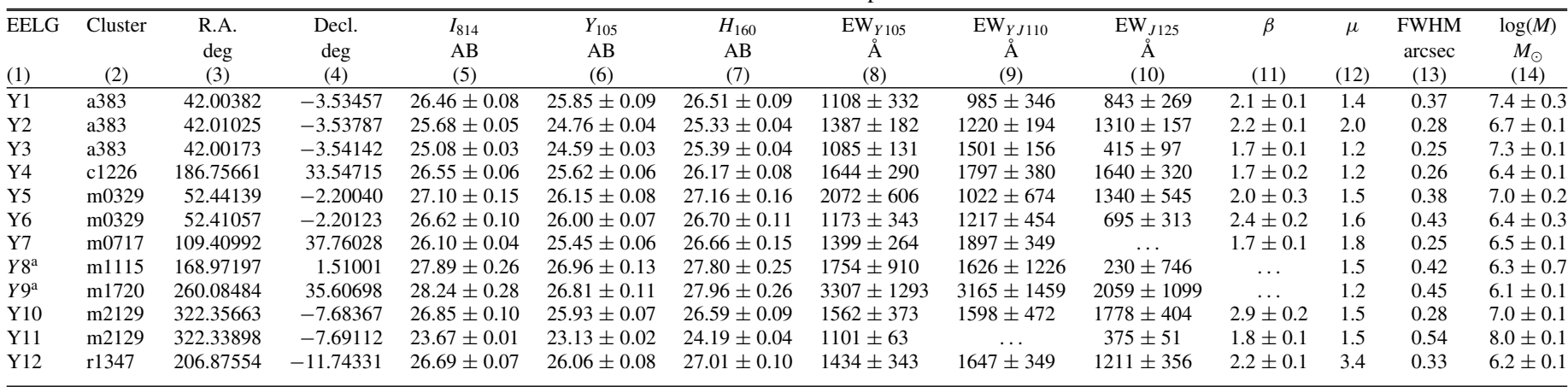

Notes. As Table 1. However, Columns (5)-(7) are apparent magnitudes for $I_{814}, Y_{105}, H_{160}$, and (8)-(10) are EWs derived from the flux excesses in $Y J_{110}, J_{125}$, and $J H_{140}$.

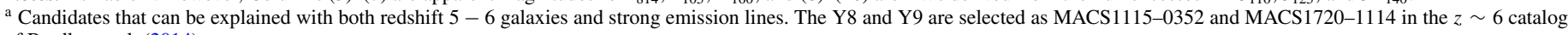
of Bradley et al. (2014).

Other emission lines, especially $\mathrm{H} \alpha \lambda 6563$ and $[\mathrm{O}$ II] $\lambda 3727$, may also contribute to the broadband photometry, but the relative flux to $\left[\mathrm{O}_{\mathrm{III}}\right] \lambda 5007$ will vary due to the difference in metallicity, star formation history, and extinction (Anders et al. 2003; Kewley et al. 2004; Salzer et al. 2005). In order to show the boosting effect, we build a model with line intensities from metallicity $Z=0.02 Z_{\odot}$ galaxy (green dots in Figure 4). In another model, we remove all other emission 

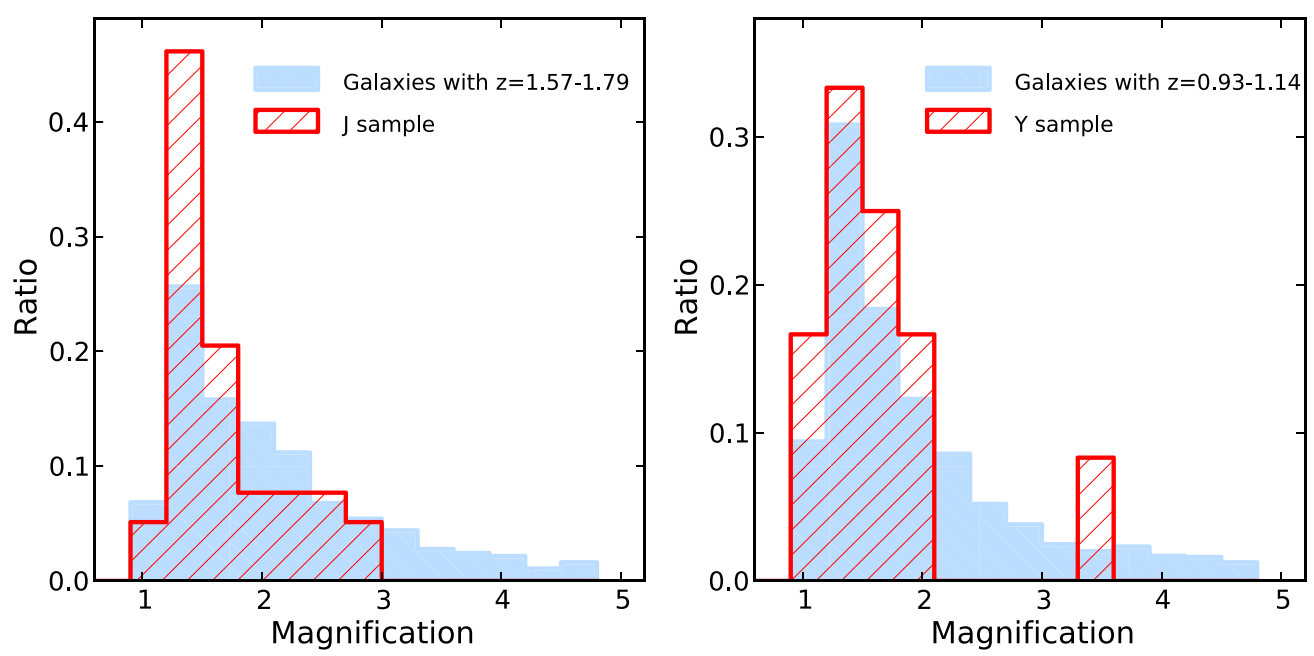

Figure 5. Magnification distributions of our $\mathrm{J}$ and $\mathrm{Y}$ samples (red histograms) and galaxies in the same redshift ranges from the cluster fields (blue filled histograms).

lines to show the contribution of [O III] $\lambda \lambda 4959,5007$ only (red lines in Figure 4). While most emission lines do not affect the photometry as significantly as [O III] $\lambda \lambda 4959,5007, \mathrm{H} \alpha \lambda 6563$ also boosts the magnitudes, as its strength is similar to [O $\mathrm{III}]$ in typical star-forming galaxies, and the $\left[\mathrm{O}_{\mathrm{II}}\right] \lambda 3727$ cannot be ignored.

As shown with the red lines in Figure 4, EELGs with redshift 1.14-1.57 would also be selected into the $\mathrm{Y}$ sample or J sample, but only if $\mathrm{H} \alpha \lambda 6563$ is extremely weak. The [O III] $\lambda 5007$ lines can be excited by massive stars as well as active galactic nuclei (AGNs). The relatively strong [O III] $\lambda 5007$ and weak $\mathrm{H} \alpha$ $\lambda 6563$ can be due to either metal-poor star-forming galaxies or the contribution of AGNs (Kauffmann et al. 2003). To exclude these EELGs from the $\mathbf{J}$ sample is not possible with current observations. In the $\mathrm{Y}$ sample, these EELGs can be identifiable by comparing the magnitudes of $J H_{140}$ and $H_{160}$. As the $\mathrm{H} \alpha$ $\lambda 6563$ falls in both the two bands in these galaxies, the two bands should be observed with similar magnitudes as shown in the left of Figure 4 . We find 5/12 candidates in the $Y$ sample have similar $J H_{140}$ and $H_{160}$ magnitudes. If parts of these weak $H_{\alpha}$ EELGs with low $H_{\alpha} /\left[\mathrm{O}_{\mathrm{III}}\right]$ ratios are AGNs, the upper limit of the AGN fraction in our Y sample is about $42 \%(5 / 12)$, which is still consistent with the AGN fraction $(\sim 17 \%)$ estimated with a larger spectral emission-line galaxy sample in Atek et al. (2011). As our analysis is not sensitive to the redshift, we assume that the redshift ranges are $z \sim 1.57-1.79$ and $0.93-1.14$ for the $\mathrm{J}$ and Y samples, respectively.

\subsection{Magnification}

The major advantage of CLASH observations is that cluster lensing provides a powerful tool to enable us to discover intrinsically faint galaxies. The magnification maps for all 25 clusters are made based on the strong lensing model of Zitrin et al. $(2009,2011)$. As magnification factors are redshiftdependent, we use the median redshift 1.03 for the $\mathrm{Y}$ sample and 1.68 for the $\mathrm{J}$ samples in the calculations. However, the following EW calculations are based on the color excesses and are thus independent of magnifications. The uncertainties of magnifications are lower than $10 \%$ for the ranges of redshifts. The estimated magnifications are likely consistent with the true value at $68 \%$ confidence for magnifications lower than 5 (Figure 11 in Bradley et al. 2014). The uncertainties are only significant for those highly magnified candidates.
The source magnification factors are listed in Tables 1 and 2. Candidate $\mathbf{J} 22$ has a magnification as large as 8.4 and shows an apparent extended structure. The delensed magnitude is estimated as $\sim 28 \mathrm{mag}$. Its $\mathrm{EW}$ is higher than $2000 \AA$, as confirmed with the color excess in $Y J_{110}, J_{125}$, and $J H_{140}$. The magnification distributions of our two samples are shown in Figure 5 in red color. We also calculate the magnifications for field galaxies that are within the same two redshift ranges as our samples in all 25 clusters and show the distributions in filled blue histograms.

\section{EW ESTIMATES}

Due to the lack of deep near-infrared spectroscopic data for our samples, we estimate the EWs by comparing the flux excess between the bands boosted by the strong emission lines (the central peak bands) and the adjacent bands dominated by the continuum (the continuum bands). As shown in Figures 3 and 4, the strong emission lines boost more than two bands; thus the combination of EWs estimated from different boosted bands makes the EW more reliable.

\subsection{Method}

The EW is estimated using two continuum bands and one central peak band through

$$
\mathrm{EW}=\frac{F_{\text {total }}-F_{c}}{F_{c}} \frac{W}{1+z},
$$

where $F_{\text {total }}$ is the flux of the central peak band, which is the total flux of emission lines and continuum, and $F_{c}$ is the continuum flux in the $I_{814}$ and $H_{160}$ bands. $W$ is the effective width of the central peak bands. $z$ is the redshift of the EELGs and is set to the median redshifts, which are 1.03 and 1.68 for the $\mathrm{Y}$ and $\mathrm{J}$ samples, to translate EW to the rest frame. An accurate continuum analysis is important to estimate the EW. In the procedure, we assume the spectral slopes equal two to estimate the continuum based on the $I_{814}$ and $H_{160}$ bands, and use the weighted averages as the continuum at the central peak bands. In the redshifts of our two samples, the rest-frame UV continuums are observed by the ACS/WFC bands; thus the UV slopes can be obtained by linear fits of these bands (Column 11 in Tables 1 and 2). The average slopes of the $\mathrm{J}$ and $\mathrm{Y}$ samples are $2.06 \pm 0.02$ and $2.07 \pm 0.03$, consistent with our assumption 

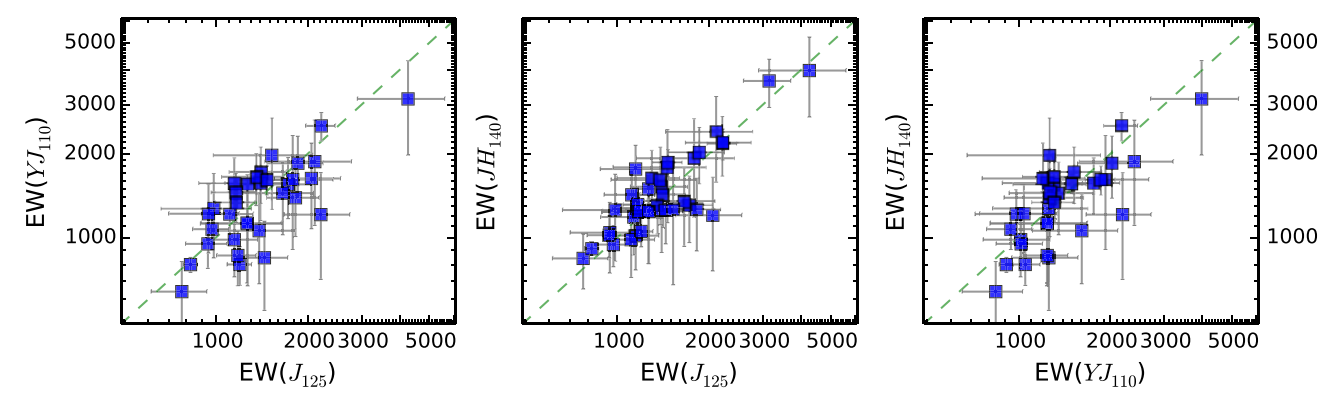

Figure 6. Comparison of EWs derived from the color excesses in three different bands for the $\mathrm{J}$ sample with the method described in Section 3.1. In these calculations, the continua are derived from $Y_{814}$ and $H_{160}$, but the EWs are derived from the excesses of $Y J_{110}, J_{125}$, and $J H_{140}$, respectively. The widths of $Y J_{110}$ and $J H_{140}$ are broader than $J_{125}$; thus the EWs derived from these two bands involve larger uncertainties. The consistency between the EWs suggests the robustness of the EW measurements.

and other studies of strong emission-line galaxies (van der Wel et al. 2011, 2013).

The uncertainties in the EW measurement are due to the $I_{814}$ and $H_{160}$ photometry and cause an overestimate of the continuum level and subsequently an underestimate of the EW. Within our model of $\mathrm{EW}=2000 \AA$, the continuum is boosted by less than $0.1 \mathrm{mag}$ for the J sample but larger for the Y sample, in which the [O II] $\lambda 3727$ line falls into the $I_{814}$ bands. Therefore, the EW should be considered as a lower limit in this situation. Furthermore, the continuum bands are considerably fainter than the central peak bands. Even if the continuum flux is estimated by averaging two continuum bands, the continuum errors are still the main factors affecting the EW measurements. For some faint EELGs, their continuum flux cannot be well constrained by photometric data due to these facts, which limit the accuracy of EW calculations.

As shown in Figures 3 and 4, all the $Y J_{110}, J_{125}$, and $J H_{140}$ are covered by the $[\mathrm{O} \mathrm{III}]+H_{\beta}$ for the $\mathrm{J}$ sample; thus the EWs can be constrained from the color excesses of these three bands. The measurements can then be verified by comparing EWs calculated from different central peak bands (Figure 6). The uncertainties in EW measurements are slightly higher for broader bands such as $Y J_{110}$. Nonetheless, the consistency of EWs inferred from three broad bands makes our results robust.

At the redshift of the Y sample (Figure 4), $\mathrm{H} \alpha \lambda 6563$ has moved into the wavelength ranges of $J_{125}$ and $J H_{140}$; both [O III $]+H_{\beta}$ and $\mathrm{H} \alpha \lambda 6563$ are covered by the $Y J_{110}$. The $Y_{105}$ band is boosted by $[\mathrm{O} \mathrm{III}]+H_{\beta}$ only. The EWs derived from the color excess are included in Table 2.

\subsection{EELGs with Extremely High EW}

In the $\mathrm{J}$ sample, the magnitudes in $Y_{105}, Y J_{110}$, and $J H_{140}$ are all boosted by [O III] $\lambda \lambda 4959,5007$, and the derived EWs can be averaged with the errors as weights to make a better constraint. Two EELGs (J2 and J29) display exceptionally large EWs of $3737 \pm 726 \AA$ and $3332 \pm 439 \AA$, respectively. As seen in Figure 7, both of these EELGs are extremely compact. The FWHM of the instrument point-spread function (PSF) is $0^{\prime \prime} .14$ for the $J_{125}$; hence the EELGs are all resolved. After subtracting the instrument PSF and the lensing effect, the measured sizes correspond to physical sizes of about 1.7 and $0.9 \mathrm{kpc}$.

In addition, the EW of Y9 is also measured to be $3307 \pm$ $1293 \AA$. As only the $Y_{105}$ is free from contaminations by other emission lines in the Y sample, the EW can only be derived from the excess of the $Y_{105}$ band and thus contains higher uncertainty. Y9 is also selected as a LBG in Bradley et al. (2014) (B13 hereafter), and we will discuss this candidates in the next section.
The same selection method as our $\mathbf{J}$ sample is also utilized for the CANDELS field in VDW11, in which 69 candidates were identified in a total area of $279 \mathrm{arcmin}^{2}$. The typical area coverage over each cluster field in CLASH is $\sim 4 \operatorname{arcmin}^{2}$, indicated from the area available within the WFC3/IR field of view. To count for the influence of the bright galaxies in the fields, we counted the areas covered by galaxies based on the segmentation images and subtracted these areas from the total areas indicated from the exposure time images. Then the total areas to search EELGs in CLASH fields is $\sim 112 \operatorname{arcmin}^{2}$. The VDW11 used the data from the Ultra Deep Survey (UDS) field in the wide program and the data from the GOODS-South Deep (GSD) field at four-epoch depth in the deep program. The VDW11 sample includes 40 and 29 EELGs from these two fields, named as the UDS sample and GSD sample hereafter. The number density for the $\mathrm{J}$ sample is $0.36 \mathrm{armin}^{-2}$, compared to $0.19 \mathrm{armin}^{-2}$ in the UDS sample and $0.39 \mathrm{armin}^{-2}$ in the GSD sample. The high detection rates in the $\mathrm{J}$ sample and the GSD sample are due to the deeper observations. As shown in Figure 8, the $\mathrm{J}$ sample is the deepest and can reach about 28.5 mag after correcting the lensing effect. The lensing effect increases the depth and reduces the volume of observations in the meantime, in addition of the small samples, we do not find significant different detection rate between the CLASH fields and the GSD field.

It is apparent in Figure 8 that the EELGs with higher EWs are more common in fainter EELGs. The three EELGs with extremely high EWs are fainter than most candidates. In the VDW sample, only two candidates have EWs larger than $2000 \AA$, which are $2304 \pm 515 \AA$ and $2002 \pm 849 \AA{ }^{22}$ Both of these candidates are selected from the GSD field, which is deeper than the UDS field. All these samples support the idea that we can detect EELGs with stronger emission lines in deeper observations. The three extreme candidates are unlikely due to noise fluctuations. We examine the probability that one EELG with $\mathrm{EW}\left(\left[\mathrm{O}_{\mathrm{III}}\right]+\mathrm{H} \beta \lambda 4861\right)=2000 \AA$ at the same redshift is measured to be $E W \geqslant 3000 \AA$. In our spectral model, fluctuations with the level of $1 / 3$ continua, which are the worst cases in our samples, are added to each band. We simulate the photometry for 10,000 times and measure the EWs with the same method. The possibility of spurious large EW $(\geqslant 3000 \AA)$ is lower than $20 \%$. The rate will be reduced to lower than $4 \%$ if the EW are measured with flux excesses in two bands, and the rate will be negligible if there are three

22 VDW11 used the EW([O III $] \lambda 5007)$ instead of $\mathrm{EW}\left([\mathrm{O} \mathrm{III}]+H_{\beta}\right)$ in their table. The EW([O III $] \lambda 5007)$ were calculated by assuming a fixed flux ratio of $H_{\beta}$ and [O III $] \lambda \lambda 4959,5007$. Therefore, we obtain the $\mathrm{EW}\left([\mathrm{O} \mathrm{III}]+H_{\beta}\right)$ using the same ratios. 

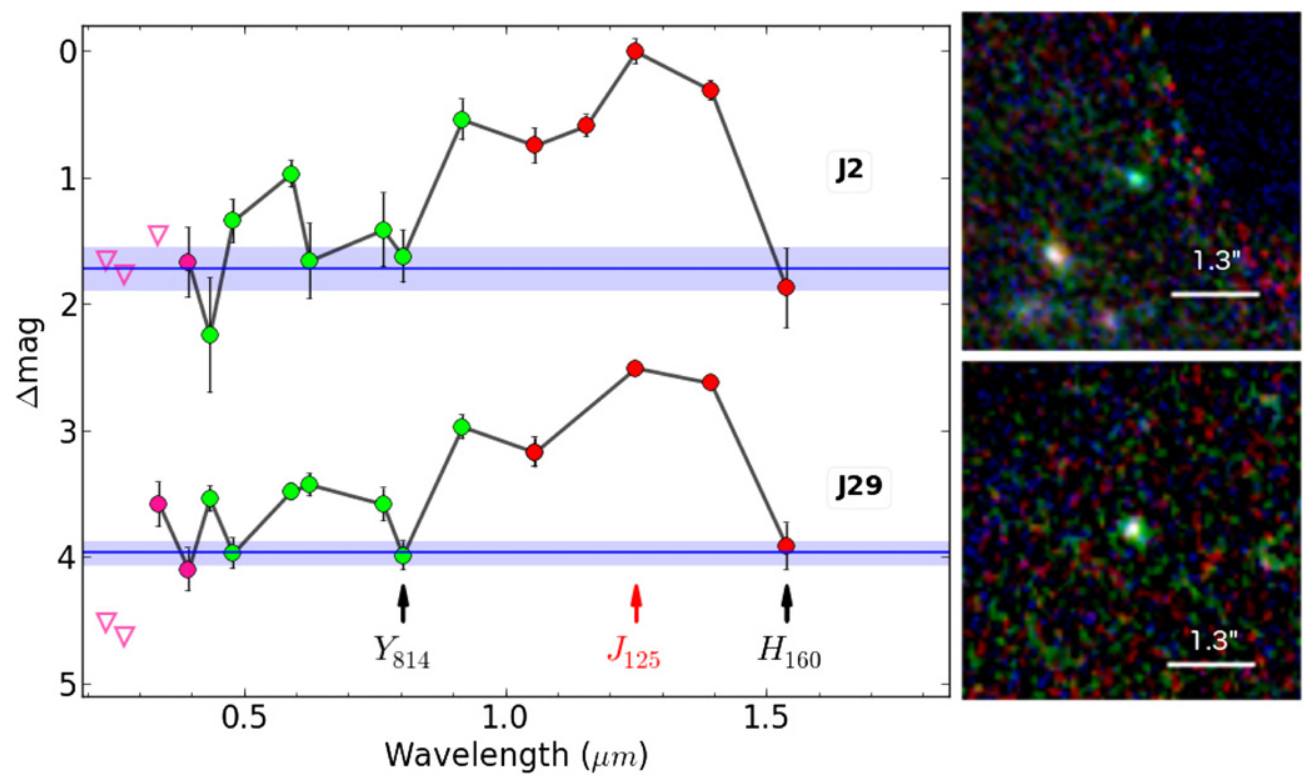

Figure 7. SED for the two EELGs with EW $3000 \AA$ in the J sample (pink, WFC3/UVIS bands; green, ACS; and red, WFC3/IR). The black and red arrows show the central wavelengths of $I_{814}, J_{125}$, and $H_{160}$ that are used in our selection. The solid blue lines are the estimated continuum levels based on the $I_{814}$ and $H_{160}$, and the shaded regions show the $1 \sigma$ error. The source fluxes are remarkably boosted by strong emission lines up to $1.5 \mathrm{mag}$. The composite color images on the right are created with $I_{814}$ and $Y_{105}$ (blue); $Y J_{110}, J_{125}$, and $J H_{140}$ (green); and $H_{160}$ (red).

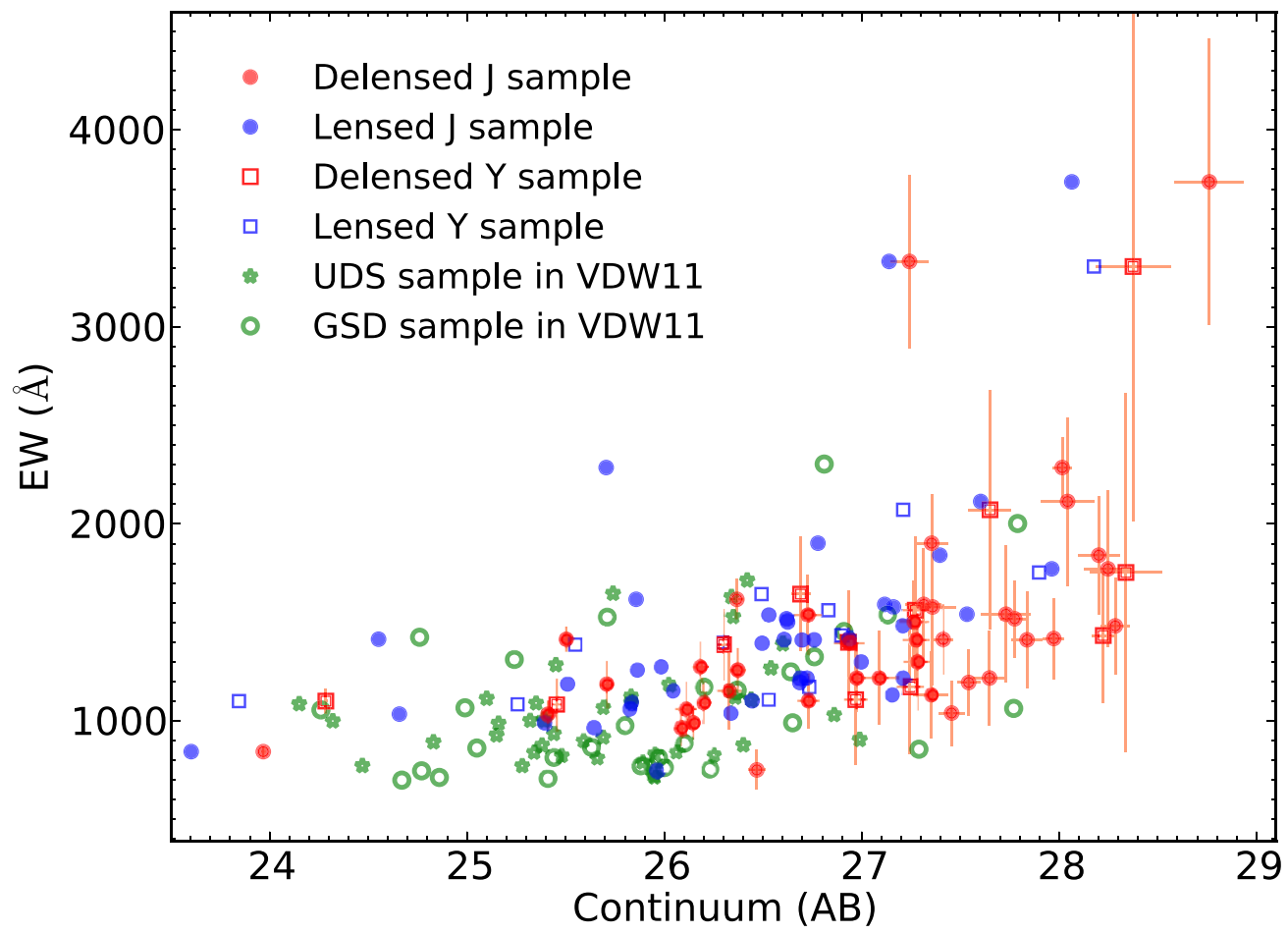

Figure 8. EW vs. continuum luminosity for EELGs in the $\mathrm{J}$ and $\mathrm{Y}$ samples and the UDS and GSD samples from VDW11. The J and Y samples are marked with filled circles and open squares, in which the observed magnitudes are shown in blue color and the intrinsic (delensed) values are shown in red color. As a comparison, the UDS and GSD samples are plotted with green stars and circles. EWs in the J sample are the weighted averages of the three EWs calculated from three different bands. EWs in the Y sample are calculated with the excesses in the $Y_{105}$ only.

bands to constrain the EW. As the spectra in Figure 7 and the results in Table 1 show, the large EWs are confirmed with three bands for the J2 candidate and two bands for the J29 candidate. Both the two EELG candidates show similar spectral shapes; moreover, the significant boosts in $Z_{850}$ and $Y_{105}$ due to the $\left[\mathrm{O}_{\text {II }}\right] \lambda 3727$ line are also in agreement with the strong [O III] $5007 \AA$ line. The EW of the Y9 candidate can only be estimated with the boost of $Y_{105}$; therefore, the noise fluctuation cannot be totally excluded, and the strength could be confirmed with further observations.

VDW11 found these candidates are low-mass $\left(\sim 10^{8} M_{\odot}\right)$, starbursting $\left(\sim 5 M_{\odot} \mathrm{yr}^{-1}\right)$, young $(5 \sim 40 \mathrm{Myr})$ galaxies. Their nature is also consistent with the spectral observations of two highly lensed EELGs at $z=1.85$ and 3.12 (Brammer et al. 2012; van der Wel et al. 2013, respectively). We estimate the age and stellar mass following the same method as VDW11. 
In general, we use the Starburst99 model (SB99, Leitherer et al. 1999) with continuous star formation and a Chabrier (2003) IMF with a high-mass cutoff at $100 M_{\odot}$ and metallicity $0.2 Z_{\odot}$. The $\operatorname{EW}\left(H_{\beta}\right)$ declines as time and is used as an age indicator. $\mathrm{EW}\left(H_{\beta}\right)$ is assumed to contribute $1 / 8$ to the combined EW. The upper limit of $\mathrm{EW}\left([\mathrm{O} \mathrm{III}]+H_{\beta}\right)$ is $4336 \AA$ from this model and will decrease to $3838 \AA$ if we use a solar metallicity. VDW11 estimated stellar masses based on the rest-frame $V$ band luminosity from the $H_{160}$ band photometry. To increase the accuracy, we use the weighted average photometry of the continue bands instead. The inferred masses are shown in Tables 1 and 2 . The median value of $7.1 \times 10^{6} M_{\odot}$ is one order lower than the VDW sample. Maseda et al. (2013) found the dynamic mass and young stellar mass ratios of the VDW sample to be similar to that of other star-forming galaxies in $z \sim 2$ and confirmed that the low stellar masses are dominated by the intense starbursts. These EELGs provide insight into the evolution of the dwarf galaxies and provide evidence that the starburst phase plays a key role in the mass buildup for at least some low-mass galaxies.

\section{DISCUSSION}

Strong emission lines are known to have a significant impact on the SED fitting (Atek et al. 2011; Schaerer \& de Barros 2009; Labbé et al. 2010, 2012; Stark et al. 2013; Shim et al. 2011; Fumagalli et al. 2012). Labbé et al. (2012) investigated the ultradeep Spitzer/IRAC photometry for a sample of $z \sim 8$ galaxies from the Hubble UDF program and found an average contribution of $\sim 0.44$ mag to the [4.5] band of Spitzer by [O III] $\lambda \lambda 4959,5007$. Schaerer \& de Barros (2009) found that the apparent Balmer breaks observed in a number of $z \sim 6$ galaxies detected at $>3.6 \mu \mathrm{m}$ with Spitzer/IRAC can be explained by the presence of redshifted strong emission lines. Smit et al. (2014) selected galaxies at narrow redshift range $z \sim 6.6-7.0$ to avoid contamination of other emission lines and detected very high [O III $]+\mathrm{H}_{\beta}$ lines. The mean value of $E W\left([\mathrm{O} \mathrm{III}]+\mathrm{H}_{\beta}\right)$ derived from the excesses of the $3.6 \mu \mathrm{m}$ band is greater than $637 \AA$ with one extreme EW of $1582 \AA$. This is consistent with the finding of Shim et al. (2011) that the fraction of emission-line galaxies evolves with redshift and that emission-line galaxies could be more common in high redshifts. However, properly considering the impact is still a challenge due to the lack of knowledge of such galaxies. The EELG samples we discovered are the median-redshift analogs and provide a good opportunity to study the high-redshift star-forming galaxies.

While emission lines have been considered in galaxy templates (Schaerer \& de Barros 2009; Ono et al. 2010), the highredshift galaxy selection itself can also be affected due to the existence of median-redshift EELGs. The fainter and stronger EELGs in our samples indicate that the impact of EELGs to the selection of LBGs has probably been underestimated. The properties of LBGs could be misunderstood due to the mix of these EELGs. Taniguchi et al. (2010) investigated the EELG interlopers for $z \sim 8$ galaxies in the Hubble Ultra Deep Field. The EWs of [O III] $\lambda 5007$ in their models only vary up to $\sim 1000 \AA$; therefore they claimed a negligible probability for low-redshift interlopers. Considering the EELGs with EW $\left([\mathrm{O}\right.$ III $\left.]+H_{\beta}\right) \geqslant 3000 \AA$ in our sample and the strong [O III] $\lambda 5007$ and $\mathrm{H} \alpha \lambda 6563$ in other surveys (Cardamone et al. 2009; Atek et al. 2011; Brammer et al. 2012; Shim \& Chary 2013; van der Wel et al. 2013; Smit et al. 2014), it is necessary to reexamine the impact of EELGs on the selections of LBGs.

\subsection{Contamination to the Selection of $z \sim 6$ Galaxies}

First, we test whether any sources in our samples would satisfy the color selections for LBGs. From Figure 1, both the terms of $J_{125}-I_{814}$ in the $\mathrm{J}$ sample and $Y_{105}-I_{814}$ in the Y sample can reach more than $1 \mathrm{mag}$. In the color-color selections of LBGs, a decrement of 1 mag is adopted (Bouwens et al. 2012; Zheng et al. 2012b; Oesch et al. 2012, 2013). Furthermore, a candidate at $z>7$ must not be detected in the optical bands. For the bright EELGs in our samples, their continuum from nearinfrared to UV bands is detected with high confidence. However, there are still a few EELGs with faint continua, and the bands bluer than $I_{814}$ fall bellow the detection limit, resulting in mimic LBGs with redshift around six. When the signal-to-noise ratio is low, it becomes difficult to distinguish whether the color excess is due to the Lyman break, as seen in LBGs, or the boost by strong emission lines in EELGs.

Recently, B13 reported a considerable number of galaxy candidates at $z \sim 6-8$ in 18 CLASH clusters (A1423, A209, CLJ1226.9+3332, MACS 0429.6-0253, MACS 1311.0-0310, MACS 1423.8+2404, and RXJ 2129.7+0005 are not included compared to the total of 25 clusters). Their selections are based on the redshifts calculated from the Bayesian photometric redshift (BPZ) code (Benítez 2000). This method also identifies high-redshift galaxy candidates primarily based on the Lyman break feature, and the results are generally in very good agreement with the common color-color selection method. Possible contaminations of EELGs at the high-redshift samples are shown by matching the samples to our EELGs. Two EELGs, Y8 and Y9, are also selected as $\operatorname{m1115-0352}(z=6.2)$ and m1720-1114 $(z=5.9)$ in the $z \sim 6$ sample of B13. Another galaxy in A209 (R.A.: 22.954264, decl.: -13.611176), which is removed from the Y sample because the $I_{814}$ band detection is below $3 \sigma$, is also included for its high photometric redshift (5.89) from BPZ and is called Y0. These candidates have similar spectral shapes, as shown with solid black circles in Figure 9. The best BPZ results are shown with open orange boxes. We also fit the $I_{814}$ band and redward bands with our strong emissionline model which is shown with blue boxes. In the emission-line model, we fix the spectral slope to two and the $\mathrm{EW}\left([\mathrm{O} \mathrm{III}]+H_{\beta}\right)$ to be the value estimated with the $Y_{105}$ band excess. Emission lines with flux ratios from a $0.2 Z_{\odot}$ galaxy (Anders et al. 2003) are considered. The only two free parameters left are the redshift and the normalization. While the high-redshift assumption often fails to fit the $H_{160}$ band, our emission line model can explain the drop of flux in the $H_{160}$ bands in three galaxies. The emissionline model also overestimates the flux in the $Y_{814}$ band. The $\chi^{2}$ values are shown with the same colors in the figure. For all the three candidates, the spectra favor the emission-line models for the slightly lower $\chi^{2}$ values.

If such candidates are chosen as LBGs, the UV-continuum slopes derived from infrared bands would be misleading. We estimate the slopes for the three candidates with a linear-fitting method for the bands redder than the $I_{814}$ band. The derived UV slopes are $3.7 \pm 0.5,3.6 \pm 0.5$, and $3.5 \pm 0.4$ for $\mathrm{Y} 0$, Y8, and Y9, respectively. Bouwens et al. (2010) obtain the UV-continuum slopes for redshift-6 galaxies with $Y_{105}, J_{125}$, and $H_{160}$. Using the same method, we get even bluer slopes, which are $4.5 \pm 0.8,4.9 \pm 0.9$, and $4.4 \pm 0.6$. These values are all at least $1 \sigma$ bluer than the mean UV-continuum slope in redshift 6 (Bouwens et al. 2010). Nevertheless, the current CLASH data and Spitzer/IRAC data are not deep enough to confirm the nature of these galaxies. Our EELG color-color selections are limited by the luminosity of the continuum. Many 


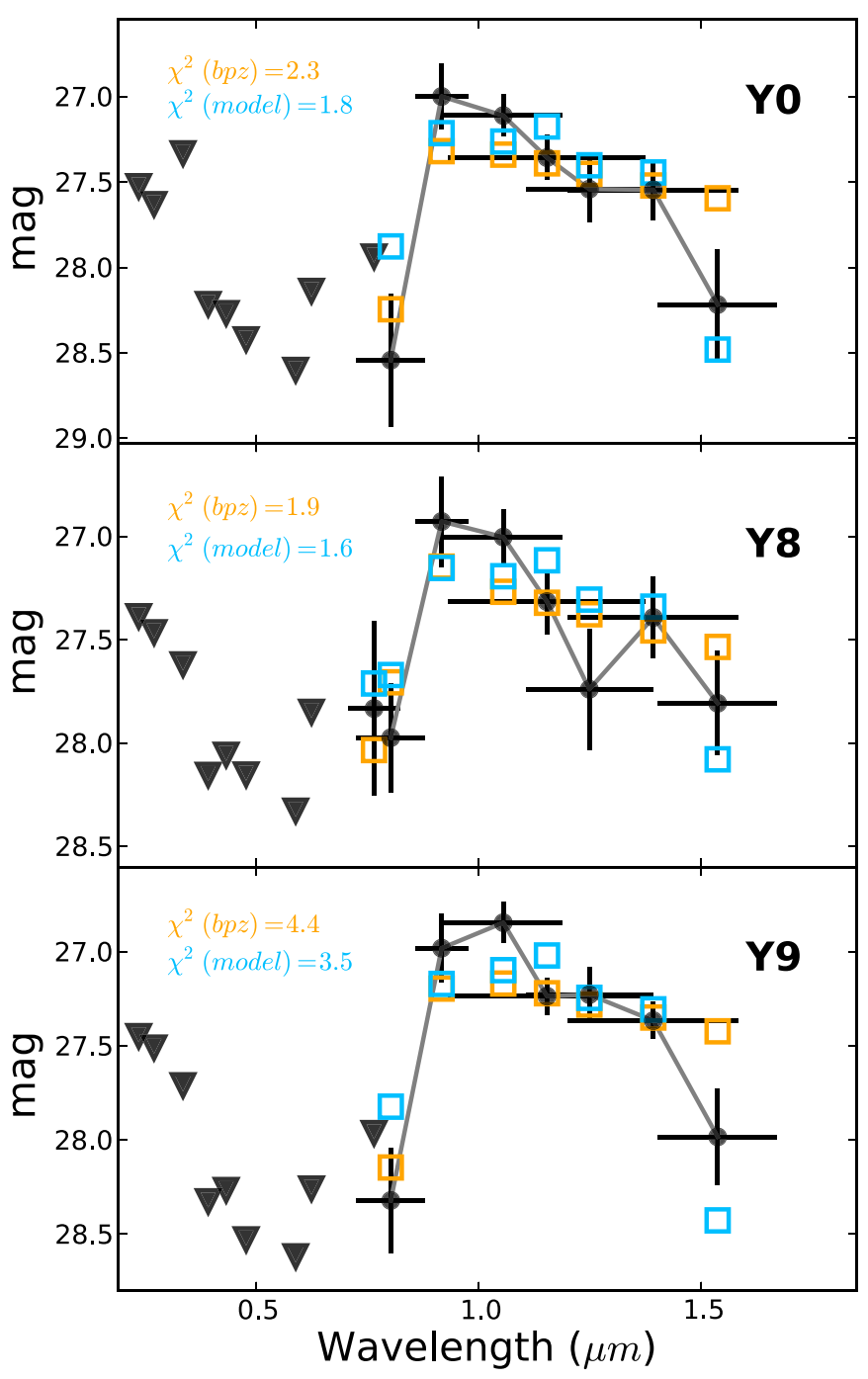

Figure 9. Observed magnitudes and best-fit SED of three EELG candidates that can also be selected out as high-redshift LBGs. The observed photometries are shown with black circles, and the triangles represent the $2 \sigma$ detection limits. All four objects display color excesses near the $Y_{105}$, which can be fit with both the LBG model (orange open squares) and the EELG model (blue open squares). In the EELG model, the spectral slope is fixed to two, and $\mathrm{EW}\left([\mathrm{O} \mathrm{III}]+H_{\beta}\right)$ is fixed to the value estimated from the color excesses in $Y_{105}$. Other metal emission lines with the flux ratios of stellar metallicity with $0.2 Z_{\odot}$ (Anders et al. 2003) have been added. The only two free parameters in the models are the redshift and normalization factor.

more EELGs with stronger emission lines are faint and below our detection limit, according to the EW and luminosity trend. What is more, our samples only include EELGs in specific redshift ranges. Therefore, the total contamination of EELGs to the high-redshift sample of B13 can be higher.

\subsection{Impact on the Selection of $z \sim 10$ Galaxies}

Recently, the search for LBGs has reached $z \sim 10$ (Zheng et al. 2012b; Bouwens et al. 2013; Ellis et al. 2013; Coe et al. 2013; Oesch et al. 2013). One of them, UDFj-39546284, has already been found to be possibly an EELG at redshift $\sim 2$ (Brammer et al. 2013; Capak et al. 2013). More candidates are found in the ongoing Hubble Frontier Fields (Zheng et al. 2014; Zitrin et al. 2014; Ishigaki et al. 2014). As there are not enough spectral observations to confirm the redshifts, properly considering the contamination of EELGs is quite necessary for these samples.
For galaxies with $z \sim 8.7-10.5$, the Ly $\alpha$-break shifts into the $J H_{140}$ band and could be selected as $J_{125}$ drop out. If the redshift is higher than 10.5 , the Ly $\alpha$-break has shifted out of the $J_{125}$ band, and only $H_{160}$ and parts of $J H_{140}$ cover the continuum. EELGs with strong emission lines falling in the $H_{160}$ band can also mimic such a spectral feature. Considering a power-law spectrum with $\mathrm{EW}\left([\mathrm{O}\right.$ III $\left.]+H_{\beta}\right)=2000(3000) \AA$ in our model, the dropout $J_{125}-H_{160} \sim 1.1(1.4)$ would be observed for EELGs at redshift 1.9. Taking into account the deviation of the slopes, which would increase the observed color difference in EELGs, we propose that, unlike current HST WFC3/IR surveys in CLASH or UDF12, at least 1 mag deeper observations in the bluer bands will be required to constrain the flux of the continuum and draw a distinction between these two scenarios. In EELGs at the matched redshift, the $\mathrm{H} \alpha \lambda 6563$ has moved out of the $H_{160}$ band, but the impact of [O II] $\lambda 3727$ on $J_{125}$ cannot be neglected; therefore the deeper observations in the $Y_{105}$ or $Y J_{110}$ bands will be helpful. If a deeper observation is not available, a significant detection in Spitzer observation is required to confirm the high-redshift LBGs (Zheng et al. 2012b; Oesch et al. 2013). Real high-redshift LBGs have a flat UV continuum, and the detection from the $H_{160}$ band to the midinfrared bands cannot be mimicked by any type of EELGs.

\subsection{EELG Fraction in High-redshift Samples}

While it is almost impossible to distinguish EELGs and highredshift galaxies without spectroscopic observations, we should consider that there are a certain number of EELGs in the highredshift galaxies. The spectral features of our J and Y samples are similar to the $I_{814}$ and $Y_{105}$ dropout galaxies at redshift range 5.6-8.0. The B13 sample includes a total of 206 galaxies in this redshift range, and we find two galaxies common to our EELG samples. If both of these galaxies are EELGs, the contamination fraction for the B13 sample is at least 2/206 1\%. However, the real contamination fraction could be higher due to the limit of the observations. Our EELG samples only include candidates such that the continua are detected higher than $3 \sigma$ in $I_{814}$. There are substantially fainter EELGs, which probably include stronger emission lines(See the trend in Figure 8).

Recently, the frontier of high-redshift samples are redshift higher than nine, selected based on the $J_{125}$ dropout. The fraction of EELGs in these $J_{125}$ dropout samples will be more significant than the contamination in the B13 sample. First, these $J_{125}$ dropout samples are in redshift range 8.7-10.5 and can be contaminated by EELGs in redshift range 1.8-2.2. The comoving volume of the redshift range 1.8-2.2 is 1.1 times the total volume of the $\mathrm{Y}$ and $\mathrm{J}$ samples. Meanwhile, the comoving volume of the $J_{125}$ dropout samples is 0.6 times the $I_{814}$ dropout samples. In the same projected area, there will be 1.1/0.6 1.8 times more contaminations due to EELGs. Second, the buildup of star-forming galaxies peaks at near redshift two, which means there is a substantial population of EELGs in that redshift. From the median redshift of our EELG samples 1.5 to redshift 1.9, the space density of star-forming galaxies increases by 2.3 times (Oesch et al. 2010). Moreover, the space densities of starforming galaxies decline since redshift around two (Bouwens et al. 2014). From redshift 6 to redshift 9, the space density of galaxies decreases about 10 times. All of these will result in that the EELG contamination fraction for the $J_{125}$ dropout samples will be $1.8 \times 2.3 \times 10 \approx 41$ times higher. Pirzkal et al. (2013) develops a Markov Chain Monte Carlo fitting method with the ability to accurately estimate the probability density function of the redshift for each object. After the analysis of the 
redshift 8-12 galaxy sample, they report that there is an average probability of $21 \%$ that these well-defined high-redshift galaxies are low-redshift interlopers.

Without infrared or deeper HST observations, these EELG contaminations cannot be effectively excluded. Therefore, it is necessary to properly include the contamination fraction of EELGs for the analysis of high-redshift samples. However, the contamination fraction is still largely uncertain due to the lack of observations of EELGs. The ongoing Hubble Frontier Fields program devotes a total of 560 orbits to observe four clusters along with four parallel blank fields. This initiative can reach the $5 \sigma$ magnitude limit of $\sim 28.7 \mathrm{mag}$, which is 1.2 mag deeper than the CLASH field. A systematical selection of EELGs in the Hubble Frontier Fields will help us to constrain the number density of faint EELGs and resolve the EELG contamination fraction for high-redshift galaxies.

\section{SUMMARY}

We have carried out two color-color selections to search for EELGs with $E W>600 \AA$ in 25 CLASH cluster fields. We identified two samples consisting of 40 and 12 EELGs in redshift ranges $1.55 \sim 1.79$ and $0.93 \sim 1.14$, respectively. Thanks to cluster lensing, EELGs are detected down to an intrinsic apparent magnitude of 28.5, which is significantly fainter than for other samples. Three candidates are found with extreme $E W>3000 \AA$, which are stronger than for other surveys. We found an abundant population of low-luminosity galaxies whose emission lines are considerably stronger than for other samples. These EELGs are considered as low-mass and strong starburst galaxies in the early stage. Future deep spectral observations are needed for such low-luminosity galaxies.

Strong emission lines in these EELGs can boost the broadband photometry by more than $1 \mathrm{mag}$. Such extreme emission lines will not only impact the SED fitting but also mimic the dropout feature seen in LBGs and contaminate the selections. We compare the EELGs and the LBGs selected from the CLASH data (Bradley et al. 2014) and find two common objects in our Y sample and the $z \sim 6$ galaxies. Both the EELGs and the LBGs can explain the spectral type, but the current photometric data are not deep enough to definitely distinguish between the two scenarios. We also notice that the possible contamination for future selections of galaxies at $z \sim 10$ cannot be ignored, and the contamination fraction could be significantly higher. One magnitude deeper observations in the bluer bands $\left(Y_{105}\right.$ or $\left.Y J_{110}\right)$ or the detection in midinfrared bands with Spitzer/IRAC may help us identify the real LBGs.

Future deep spectroscopic observations of EELGs such as the James Webb Space Telescope are needed to make accurate measurements of emission lines and unveil the nature of these EELGs. Furthermore, the ongoing HST observations of frontier fields provide unprecedented deep observations of six clusters and six parallel fields. ${ }^{23}$ We predict that these deep observations will reveal dozens of EELGs with possible stronger emission lines, which will help us to confirm the EW and luminosity trend and estimate the number density.

The CLASH program (GO-12065) is based on observations made with the NASA/ESA Hubble Space Telescope. The Space Telescope Science Institute is operated by the Association of Universities for Research in Astronomy, Inc., under NASA Contract NAS 5-26555. J.X.W. acknowledges support from

\footnotetext{
23 For details, see http://www.stsci.edu/hst/campaigns/frontier-fields/.
}

Chinese NSF (grant Nos. 11233002 and 11421303) and the Strategic Priority Research Program "The Emergence of Cosmological Structures" of the Chinese Academy of Sciences (Grant XDB09000000).

Facility: HST (ACS,WFC3)

\section{REFERENCES}

Anders, P., \& Fritze-v. Alvensleben, U. 2003, A\&A, 401, 1063

Atek, H., Siana, B., Scarlata, C., et al. 2011, ApJ, 743, 121

Benítez, N. 2000, ApJ, 536, 571

Bertin, E., \& Arnouts, S. 1996, A\&AS, 117, 393

Blakeslee, J. P., Anderson, K. R., Meurer, G. R., Benítez, N., \& Magee, D. 2003, in ASP Conf. Ser. 295, Astronomical Data Analysis Software and Systems XII, ed. H. E. Payne, R. I. Jedrzejewski, \& R. N. Hook (San Francisco, CA: ASP), 257

Bouwens, R. J., Illingworth, G. D., Franx, M., et al. 2009, ApJ, 705, 936 Bouwens, R. J., Illingworth, G. D., Labbe, I., et al. 2011, Natur, 469, 504 Bouwens, R. J., Illingworth, G. D., Oesch, P. A., et al. 2010, ApJL, 708, L69 Bouwens, R. J., Illingworth, G. D., Oesch, P. A., et al. 2012, ApJ, 754, 83 Bouwens, R. J., Illingworth, G. D., Oesch, P. A., et al. 2014, arXiv:1403.4295 Bouwens, R. J., Oesch, P. A., Illingworth, G. D., et al. 2013, ApJL, 765, L16 Bradley, L. D., Zitrin, A., Coe, D., et al. 2014, ApJ, 792, 76 Brammer, G. B., Sánchez-Janssen, R., Labbé, I., et al. 2012, ApJL, 758, L17 Brammer, G. B., van Dokkum, P. G., Illingworth, G. D., et al. 2013, ApJL, 765, L2

Capak, P., Faisst, A., Vieira, J. D., et al. 2013, ApJL, 773, L14

Cardamone, C., Schawinski, K., Sarzi, M., et al. 2009, MNRAS, 399, 1191

Cardelli, J. A., Clayton, G. C., \& Mathis, J. S. 1989, ApJ, 345, 245

Chabrier, G. 2003, PASP, 115, 763

Coe, D., Zitrin, A., Carrasco, M., et al. 2013, ApJ, 762, 32

Ellis, R. S., McLure, R. J., Dunlop, J. S., et al. 2013, ApJL, 763, L7

Erb, D. K., Steidel, C. C., Shapley, A. E., et al. 2006, ApJ, 647, 128

Frye, B. L., Hurley, M., Bowen, D. V., et al. 2012, ApJ, 754, 17

Fumagalli, M., Patel, S. G., Franx, M., et al. 2012, ApJL, 757, L22

Gonzaga, S., Hack, W., Fruchter, A., \& Mack, J.(ed.) 2012, The DrizzlePac Handbook (Baltimore, MD: STScI)

Grogin, N. A., Kocevski, D. D., Faber, S. M., et al. 2011, ApJS, 197, 35

Ishigaki, M., Kawamata, R., Ouchi, M., et al. 2014, ApJ, 799, 12

Kauffmann, G., Heckman, T. M., Tremonti, C., et al. 2003, MNRAS, 346, 1055

Kewley, L. J., Geller, M. J., \& Jansen, R. A. 2004, AJ, 127, 2002

Koekemoer, A. M., Ellis, R. S., McLure, R. J., et al. 2013, ApJS, 209, 3

Koekemoer, A. M., Faber, S. M., Ferguson, H. C., et al. 2011, ApJS, 197, 36

Koekemoer, A. M., Fruchter, A. S., Hook, R. N., \& Hack, W. 2003, in HST Calibration Workshop: Hubble after the Installation of the ACS and the NICMOS Cooling System, ed. S. Arribas, A. Koekemoer, \& B. Whitmore (Baltimore, MD: Space Telescope Science Institute), 337

Labbé, I., González, V., Bouwens, R. J., et al. 2010, ApJL, 716, L103

Labbé, I., Oesch, P. A., Bouwens, R. J., et al. 2012, ApJL, 777, L19

Leitherer, C., Schaerer, D., Goldader, J. D., et al. 1999, ApJS, 123, 3

Maseda, M. V., van der Wel, A., da Cunha, E., et al. 2013, ApJL, 778, L22 Oesch, P. A., Bouwens, R. J., Carollo, C. M., et al. 2010, ApJL, 725, L150 Oesch, P. A., Bouwens, R. J., Illingworth, G. D., et al. 2012, ApJ, 759, 135 Oesch, P. A., Bouwens, R. J., Illingworth, G. D., et al. 2013, ApJ, 786, 108 Ono, Y., Ouchi, M., Shimasaku, K., et al. 2010, MNRAS, 402, 1580 Pirzkal, N., Rothberg, B., Ryan, R., et al. 2013, ApJ, 775, 11

Postman, M., Coe, D., Benítez, N., et al. 2012, ApJS, 199, 25

Salzer, J. J., Lee, J. C., Melbourne, J., et al. 2005, ApJ, 624, 661

Schaerer, D., \& de Barros, S. 2009, A\&A, 502, 423

Shim, H., \& Chary, R. 2013, ApJ, 765, 26

Shim, H., Chary, R., Dickinson, M., et al. 2011, ApJ, 738, 69

Smit, R., Bouwens, R. J., Labbé, I., et al. 2014, ApJ, 784, 58

Stark, D. P., Schenker, M. A., Ellis, R., et al. 2013, ApJ, 763, 129

Storey, P. J., \& Hummer, D. G. 1995, MNRAS, 272, 41

Straughn, A. N., Pirzkal, N., Meurer, G. R., et al. 2009, AJ, 138, 1022

Taniguchi, Y., Shioya, Y., \& Trump, J. R. 2010, ApJ, 724, 1480

van der Wel, A., Straughn, A. N., Rix, H.-W., et al. 2011, ApJ, 742, 111

van der Wel, A., van de Ven, G., Maseda, M., et al. 2013, ApJL, 777, L17

Zheng, W., Bradley, L. D., Saraff, A., et al. 2012a, in Seventh Conference on Astronomical Data Analysis, http://ada7.cosmostat.org/ ADA7_proceeding_Zheng.pdf

Zheng, W., Postman, M., Zitrin, A., et al. 2012b, Natur, 489, 406

Zheng, W., Shu, X., Moustakas, J., et al. 2014, ApJ, 795, 93

Zitrin, A., Broadhurst, T., Coe, D., et al. 2011, ApJ, 742, 117

Zitrin, A., Broadhurst, T., Umetsu, K., et al. 2009, MNRAS, 396, 1985

Zitrin, A., Zheng, W., Broadhurst, T., et al. 2014, ApJL, 793, L12 